\title{
Characterization of NIR InGaAs imager arrays for the JDEM SNAP mission concept
}

S. Seshadri, D. M. Cole, B. Hancock, P. Ringold, C. Peay, et al.

S. Seshadri, D. M. Cole, B. Hancock, P. Ringold, C. Peay, C. Wrigley, M. Bonati, M. G. Brown, M. Schubnell, G. Rahmer, D. Guzman, D. Figer, G. Tarle, R. M. Smith, C. Bebek, "Characterization of NIR InGaAs imager arrays for the JDEM SNAP mission concept," Proc. SPIE 6276, High Energy, Optical, and Infrared Detectors for Astronomy II, 62760S (20 June 2006); doi: $10.1117 / 12.673252$

Event: SPIE Astronomical Telescopes + Instrumentation, 2006, Orlando, Florida , United States 


\title{
"Characterization of NIR InGaAs imager arrays for the JDEM SNAP mission concept
}

\author{
S. Seshadri*a , D.M. Cole ${ }^{\mathrm{a}}$, B. Hancock ${ }^{\mathrm{a}}$, P. Ringold ${ }^{\mathrm{a}}$, C. Peay ${ }^{\mathrm{a}}$, C.Wrigley ${ }^{\mathrm{a}}$, M. Bonati ${ }^{\mathrm{b}}$, M.G. \\ Brown $^{\mathrm{c}}$, M. Schubnell ${ }^{\mathrm{c}}$, G. Rahmer ${ }^{\mathrm{b}}$, D. Guzman ${ }^{\mathrm{b}}$, D. Figer ${ }^{\mathrm{d}}$, G. Tarle ${ }^{\mathrm{c}}$, R.M. Smith ${ }^{\mathrm{b}}$, and C. Bebek ${ }^{\mathrm{e}}$ \\ ${ }^{a} J e t$ Propulsion Laboratory 4800 Oak Grove Drive, Pasadena, CA, USA 91109, \\ ${ }^{\mathrm{b}}$ California Institute of Technology, Pasadena, CA, \\ ${ }^{c}$ University of Michigan, Ann Arbor, MI, \\ ${ }^{\mathrm{d}}$ Space Telescope Science Institute, Baltimore, MD, now with Rochester Institute of Technology, \\ Rochester, NY, \\ ${ }^{\mathrm{e}}$ Lawrence Berkeley National Laboratory, Berkeley, CA, Department of Physics,
}

\begin{abstract}
We present the results of a study of the performance of InGaAs detectors conducted for the SuperNova Acceleration Probe (SNAP) dark energy mission concept. Low temperature data from a nominal 1.7 um cut-off wavelength $1 \mathrm{kx} 1 \mathrm{k}$ InGaAs photodiode array, hybridized to a Rockwell H1RG multiplexer suggest that InGaAs detector performance is comparable to those of existing 1.7 um cut-off $\mathrm{HgCdTe}$ arrays. Advances in 1.7um $\mathrm{HgCdTe}$ dark current and noise initiated by the SNAP detector research and development program makes it the baseline detector technology for SNAP. However, the results presented herein suggest that existing InGaAs technology is a suitable alternative for other future astronomy applications.
\end{abstract}

Keywords: NIR, InGaAs, astronomy, low-temperature, focal plane array, imager, SNAP

\section{INTRODUCTION}

The SuperNova/Acceleration Probe (SNAP) mission concept requires $>150$ megapixel, $0.9-1.7 \mathrm{um}$ band Near Infrared (NIR) focal plane mosaic with high Quantum Efficiency (QE $>60 \%)$, low dark current $(<0.2 \mathrm{e} / \mathrm{sec}$ at $140 \mathrm{~K})$ and low noise $(<8 \mathrm{e}$ - for $\mathrm{n}=16$ Fowler samples and exposure time of $300 \mathrm{sec})$. The linkage between SNAP science and detector performance requirements are described elsewhere in these proceedings. ${ }^{1}$ SNAP detector R\&D goals were to find detectors able to meet nominal performance goals and to develop technology where needed. In the process detector performance goals were refined and additional criteria identified. At the start of the SNAP program, concerns over noise, dark current and QE of existing 1.7 um cut-off wavelength $\mathrm{HgCdTe}$ imagers spurred interest in InGaAs as an alternative detector technology for SNAP. Simultaneously, the SNAP R\&D effort undertook a program to improve these parameters in $\mathrm{HgCdTe}$ technology. The significant progress in these areas is described elsewhere in these proceedings. ${ }^{2,3}$

While InGaAs imagers are being considered for use in astronomical applications, ${ }^{4}$ their use is not yet widespread. InGaAs is an established detector technology with a large commercial base. Small format arrays are used in Dense Wavelength Division Multiplexing (DWDM) telecommunications applications. For example, in contrast to some early 1.7 um cut-off $\mathrm{HgCdTe}$ imagers, InGaAs detectors with high, flat QE already exist. ${ }^{5}$ A transparent InP substrate and substrate removal technology, such as that used to increase short wavelength performance and minimize cut-off wavelength non-uniformities and cosmic ray effects in $\mathrm{HgCdTe}$, also exists. There is also a developing base for large format 2-D imager arrays for military and aerospace applications. ${ }^{5,6}$ The larger commercial market for InGaAs also offers this imager technology the potential to be a lower cost, higher reliability alternative to $\mathrm{HgCdTe}$. The work described herein provides data to assess the suitability of InGaAs technology for SNAP and determine its viability for other astronomy/scientific applications.

*Email: suresh.seshadri@jpl.nasa.gov; PH: (818)-354-8370

High Energy, Optical, and Infrared Detectors for Astronomy II

edited by David A. Dorn, Andrew D. Holland Proc. of SPIE Vol. 6276

62760S, (2006) - 0277-786X/06/\$15 - doi: 10.1117/12.673252

Proc. of SPIE Vol. 6276 62760S-1 


\section{METHODOLOGY}

The InGaAs detector we tested (part \#H1RG-015) was grown (with a composition that is lattice-matched to an InP substrate) and fabricated by Sensors Unlimited, Inc. (now part of the Optical and Space Systems Division of Goodrich Corporation) in $\sim 2002$. Rockwell Scientific hybridized the detector onto their H1RG readout multiplexer such that illumination was via the backside of the detector, through the InP substrate, analogous to conventional $\mathrm{HgCdTe}$ focal plane arrays. Temperature stability and control during testing was established using a Lakeshore 332 controller which has a $1 \mathrm{mK}$ control and readout accuracy. Thermal cycling of the package during testing was controlled at a slew rate of $0.5 \mathrm{~K} / \mathrm{min}$ under normal operation with a peak of $<5 \mathrm{~K} / \mathrm{min}$ under natural (uncontrolled) dewar warm-up, in the event of control failure, to limit the stress of thermal mismatch between the package, multiplexer and detector from adversely or catastrophically affecting the detector.

The InGaAs detector was operated in the H1RG's slow readout mode, with buffered output, with an external current source. An array controller from Astronomical Research Cameras, Inc. (commonly referred to as "Generation III, Leach controller") provided clocking and bias signals. Bias levels were generated on an 8-channel analog video processor board. Two of these boards were used for 16 channel readout of the detector. The most important biases settings used in the tests are shown in Table 1. Clocks were provided on a separate digital clock driver board. With the controller driving the multiplexer clock at a rate of $110 \mathrm{kHz}$, the integration time per pixel before conversion was $1 \mu \mathrm{s}$.

\begin{tabular}{|l|c|}
\hline \multicolumn{2}{|c|}{$\begin{array}{c}\text { Table 1: H1RG analog } \\
\text { signal chain bias settings. }\end{array}$} \\
\hline Vdd (V) & 3.3 \\
\hline Vbp (V) & 3.3 \\
\hline Vbg (V) & 2.4 \\
\hline Vdsub (V) & 1.0 \\
\hline Vrst (V) & 0.5 \\
\hline
\end{tabular}

The multiplexer was read out in 16-channel output mode, each output corresponding to a 64 column, 1024 row slice of the array. This output was digitized by a 16-bit Analog-to-Digital Converter (ADC). The offset of the video board gain stage for each channel was optimized to maximize the $0.5 \mathrm{~V}$ signal swing at the detector over the entire ADC range. Software control of the detector and control electronics was established using custom software. ${ }^{7}$

All our data consist either of correlated double sample (CDS) pairs, or of series of samples taken up-the-ramp. In both cases, the detector was reset by clocking through the entire array with reset enabled; the array was then read out immediately, to create a "reset" frame. This corresponds to Rockwell Scientific's line-by-line reset (option B), which we found was the most effective at repeatably resetting pixels. This minimized data corruption by dynamic self-heating effects in the detector/multiplexer package. For the CDS data, we also clocked through the array without reading it, immediately before the exposure read, to duplicate the initial sample conditions as precisely as possible. Because artifacts were sometimes still present, we still routinely removed the first frame from our analysis to minimize any clocking-related issues.

\section{RESULTS}

\subsection{Conversion gain (and its inverse)}

The conversion gain $\left(\mathrm{C}_{\mathrm{g}}\right)$ of a detector is a fundamental parameter that relates the photo-generated charge captured at the sense node of the detector to the measured output voltage (or its digitized value) of the readout electronics. Two related definitions exist for the conversion gain. The engineering gain is typically given in units of $\mu \mathrm{V} / \mathrm{e}-$. However, astronomers often refer to conversion gain as e-/Digital Number (DN) or e-/ADU (Analog-Digital Unit). We bridge this nomenclature difference, by calling the astronomers' definition the inverse gain $\left(=1 / C_{g}\right)$. Furthermore, whether referring to the conversion gain or its inverse, the output units will be in DN (which we use interchangeably with ADU), instead of $\mu \mathrm{V}$. There are several methods to determine $\mathrm{C}_{g}$, the fundamental assumption of these approaches being that the variance of the signal level is proportional to the shot noise of the incident photons. In this instance, Poisson statistics 
dictates that the mean of the signal is directly related to the variance (and, therefore, to the square of the standard deviation), the proportionality factor being the conversion gain.

Conversion gain is usually referred to as a fixed number over the entire signal range. While this assumption is normally true for the high bias conditions used for CCDs, it is generally not true for the low-bias voltage conditions under which IR focal planes are typically operated, owing to signal-level dependent non-linearities in the signal chain (e.g. sense node capacitance, amplifiers, switches, etc.). A mean-variance curve for the InGaAs detector is shown in Figure 1a. The conversion gain calculated from this data is shown in Figure $1 \mathrm{~b}{ }^{8}$ From the figure, it is apparent that $\mathrm{C}_{\mathrm{g}}$ is signaldependent, with noticeable deviation from constancy at signal levels as low as $10-15 \%$ of full scale.

It has recently been noted that capacitive coupling of pixels can result in a correction to the conversion gain as calculated above. ${ }^{9}$ An interpixel capacitance factor of 0.87 was calculated for these detectors using a noise autocorrelation method, resulting in a $\sim 13 \%$ gain error. ${ }^{10}$ Thus, the low signal inverse gain is de-rated by this amount from $\sim 7.3 \mathrm{e}-/ \mathrm{DN}$ to $\sim 6.3 \mathrm{e}-$ /DN.
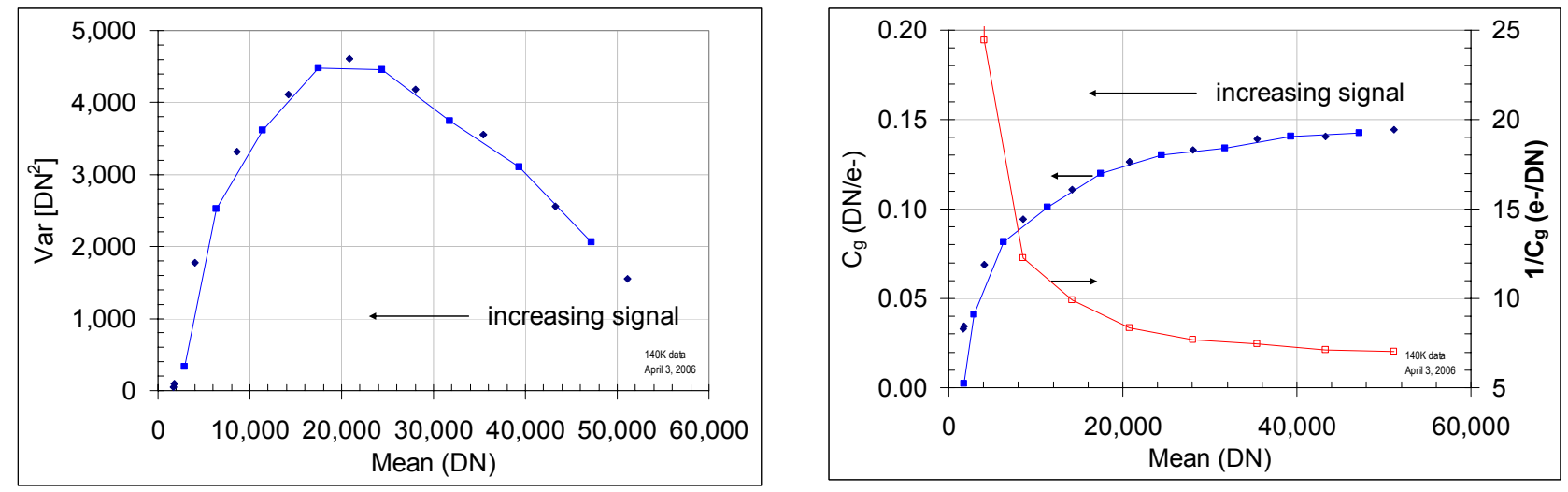

Figure 1: (a) Mean-variance and (b) conversion gain plots for an InGaAs detector at 140K. Each data point represents a spatial average of the 6 frame temporal variance from each of a $32 \times 32$ block of pixels. The temporal variance data has been adjusted to correct for sampling bias in the variance estimation.

\subsection{Linearity and full well}

The measured linearity and full well, calculated using the conversion gain data from section 3.1, are illustrated in Figure 2. The conversion gain calculation algorithm forces $\mathrm{dQ} / \mathrm{dt}$ to be constant, thereby capturing all non-linearities into a function, $\mathrm{C}_{\mathrm{g}}(\mathrm{V})$. This explains the linear charge versus time curve in the figure. A full well of $435 \mathrm{~K}$ e- is established for the $0.5 \mathrm{~V}$ detector bias.

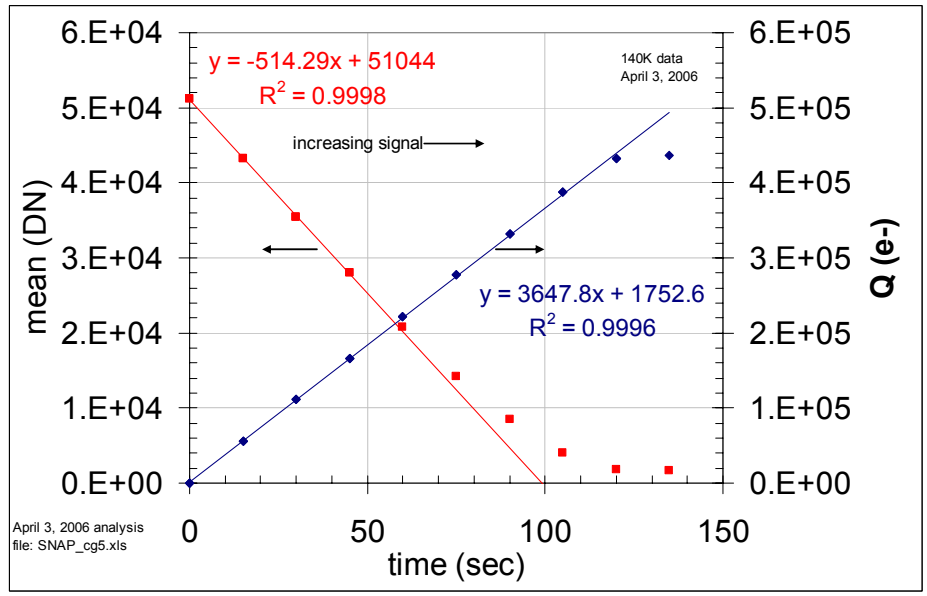

Figure 2: Linearity and full well of the InGaAs/mux hybrid package at $140 \mathrm{~K}$. The (red) line corresponds to a linear fit to the first 5 points in the mean data. 


\subsection{Quantum efficiency}

Figure 3 illustrates the relative $\mathrm{QE}$ of the InGaAs detector at 140K. A nominal room temperature curve obtained from Goodrich Corporation is also shown in the figure for comparison. It is evident that the spectral shape is retained, with the wavelength being shifted towards shorter wavelengths due to the band gap shift of the detector band edge. The shift corresponds to $\sim 0.6 \mathrm{~nm} /{ }^{\circ} \mathrm{K}$ or $\sim 0.28 \mathrm{meV} /{ }^{\circ} \mathrm{K}$, resulting in a $100 \mathrm{~nm}$ shorter cut-off than required by the original SNAP specification.

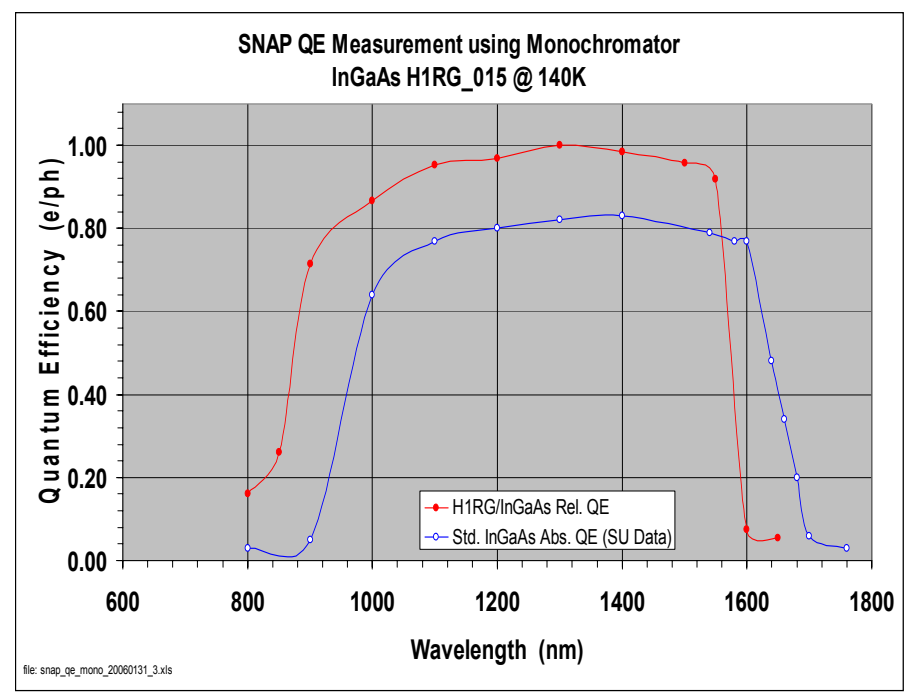

Figure 3: Relative quantum efficiency of the InGaAs detector at $140 \mathrm{~K}$.

\subsection{Dark current}

The dark current of the InGaAs detector was determined from measured sample-up-the-ramp data of a defect and artifact-free region from 8 of the 16 channels of the detector. The dark current images shown in Figure $4 \mathrm{a}$ are typical of other images from longer integration times in the sample-up-the-ramp data. The image appears to contain a fuzzy background that has been attributed to cosmic ray events in the substrate of $\mathrm{HgCdTe}$ detectors and could be the operating mechanism in this instance as well. The frame average signal level for the sample-up-the-ramp data from which the dark current is calculated is shown in Figure $4 b$.

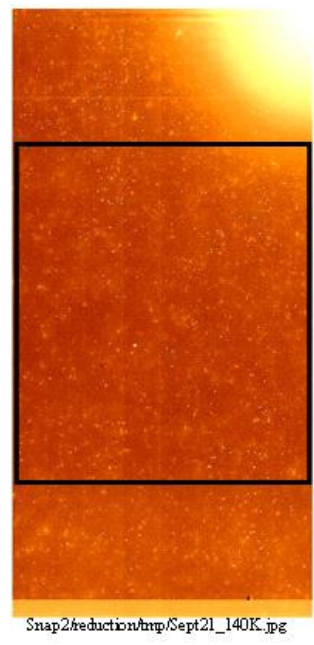

(a)

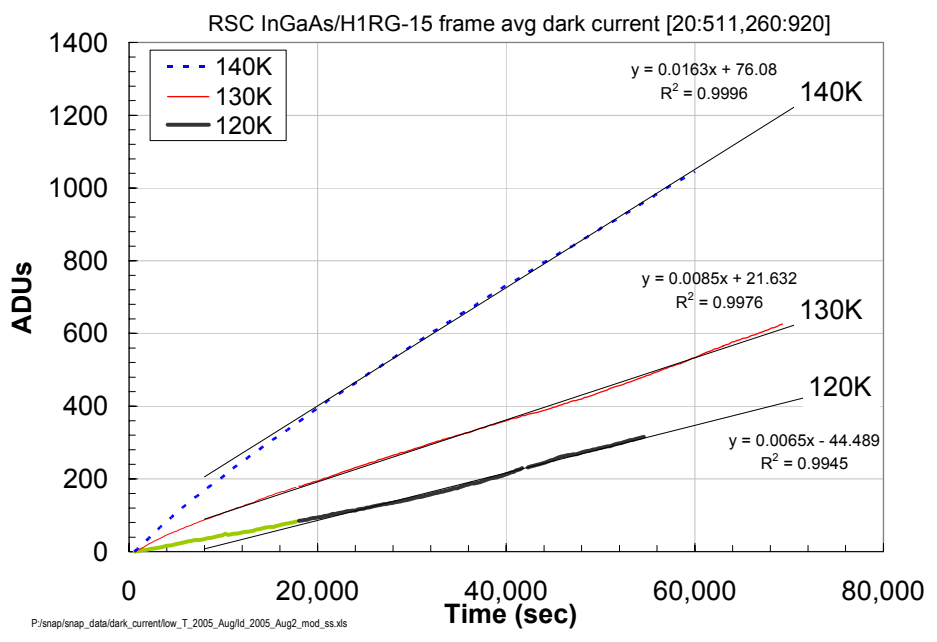

(b)

Figure 4: (a) Dark image and (b) corresponding frame averaged dark current data at varying temperatures. A hole mask (not shown) was used to verify the absence of external light leak influence on the data. The region outlined in black in part (a) was used in the analysis to avoid the influence of the light/charge-inducing defect at the top right of the figure, even at the longest integration times. 
The calculated dark current is seen to accurately represent the data, provided an $\sim 4-5$ hour stabilization time is allowed for after each temperature change. We also evaluated the dark current of each pixel from the same data set. The resulting dark current histogram of all of the pixels in the specified region is shown in Figure 5. The histograms appear to be normally distributed, with the exception of the commonly seen high dark current tails. Figure 6 illustrates an Arrhenius plot of the temperature dependence of the mean dark current. The curve is relatively well behaved, with an extrapolated value that matches nominal room temperature data. The data also indicate that the existing InGaAs detectors meet SNAP requirements within the nominal SNAP operating temperature band.

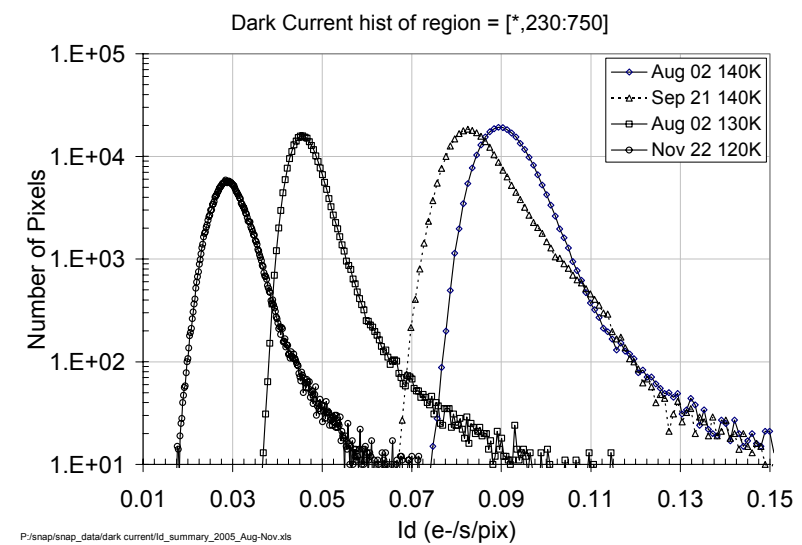

Figure 5: A histogram of the calculated dark current for pixels in the region in figure $4 \mathrm{a}$ at various temperatures.

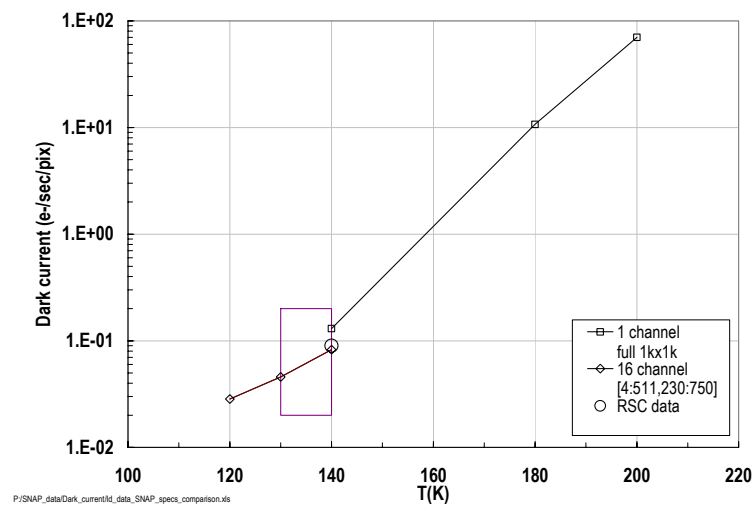

Figure 6: Arrhenius plot of InGaAs dark current.

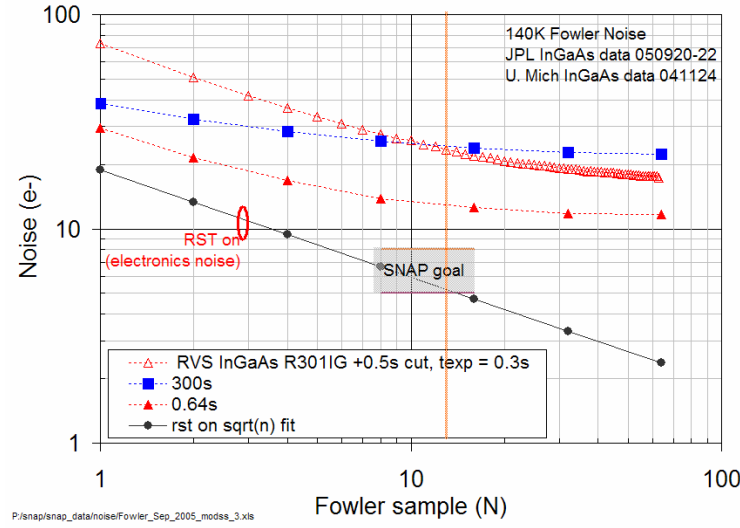

Figure 8: Noise versus Fowler sample at minimum and SNAP 300s integration intervals for InGaAs detector arrays hybridized to Rockwell H1RG and Raytheon Virgo multiplexers. Curves of the electronics noise of our setup, obtained by holding the reset switch on during the measurement is also shown for comparison.

\subsection{Noise}

Because it limits the $\mathrm{S} / \mathrm{N}$ science observations, the QE/noise parameter is a fundamental issue for SNAP detector development. While the situation has been somewhat more relaxed at present, no detector/mux combination could meet 
the original specifications of 5-8 e- noise for a Fowler 8-16 samples under the SNAP 300s integration time at the start of the SNAP R\&D program. A histogram of a CDS noise image (a dark CDS image taken at minimum integration time) with and without the reset switch on is shown in Figure 7. The variation in the two histograms reflects the excess noise due to the detector, which is seen to be normally distributed. This is similar to the experience with Rockwell HgCdTe detectors hybridized to the H1RG multiplexer. ${ }^{3}$

Figure 8 illustrates the noise performance of the InGaAs focal plane array versus sampling depth, N, the number of images averaged together to reduce uncorrelated noise effects such as photon shot noise. The JPL data was taken by locating the mean of the histogram and showed no variation with the application of different sigma clipping and hot pixel masking techniques. The University of Michigan noise data (labeled RVS InGaAs in the figure because the InGaAs was hybridized to a Raytheon Vision Systems multiplexer) was obtained by $0.5 \sigma$ clipping of their histograms. A few facts are evident from the figure. Firstly, the InGaAs detector fails to meet the original SNAP specifications. The resulting noise is also higher than that obtained from recent SNAP-developed HgCdTe arrays. ${ }^{2}$ Secondly, while the electronics noise is well behaved, decreasing in line with the expected square root dependence with increasing Fowler sample, the excess detector noise does not. We measured the correlated noise effects by comparing the average value of reference pixels in the H1RG to comparable set of pixels in the detector for each of eight of the 16 readout channels. This data, which is shown in Figure 9, illustrates that a strong degree of correlation does exist. However, attempts to minimize these effects via reference pixel subtraction did not result in a vastly improved Fowler noise curve. Thirdly, the InGaAs detector hybridized to a Rockwell multiplexer has lower noise at all Fowler samples than one hybridized to the Raytheon multiplexer, at least for the multiplexer version of each that was used. These limitations, combined with recent developments in $\mathrm{HgCdTe}$ arrays presently favor HgCdTe technology for SNAP. However, the picture for non-SNAP applications is very encouraging, especially given the fact that the existing (4 year old) InGaAs detector noise is at the same level as that for the $1.7 \mathrm{um} \mathrm{HgCdTe}$ at the start of the SNAP detector development program.

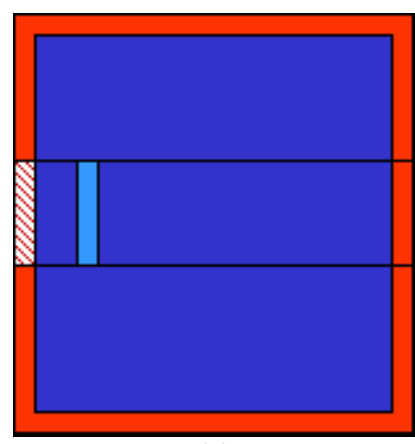

(a)

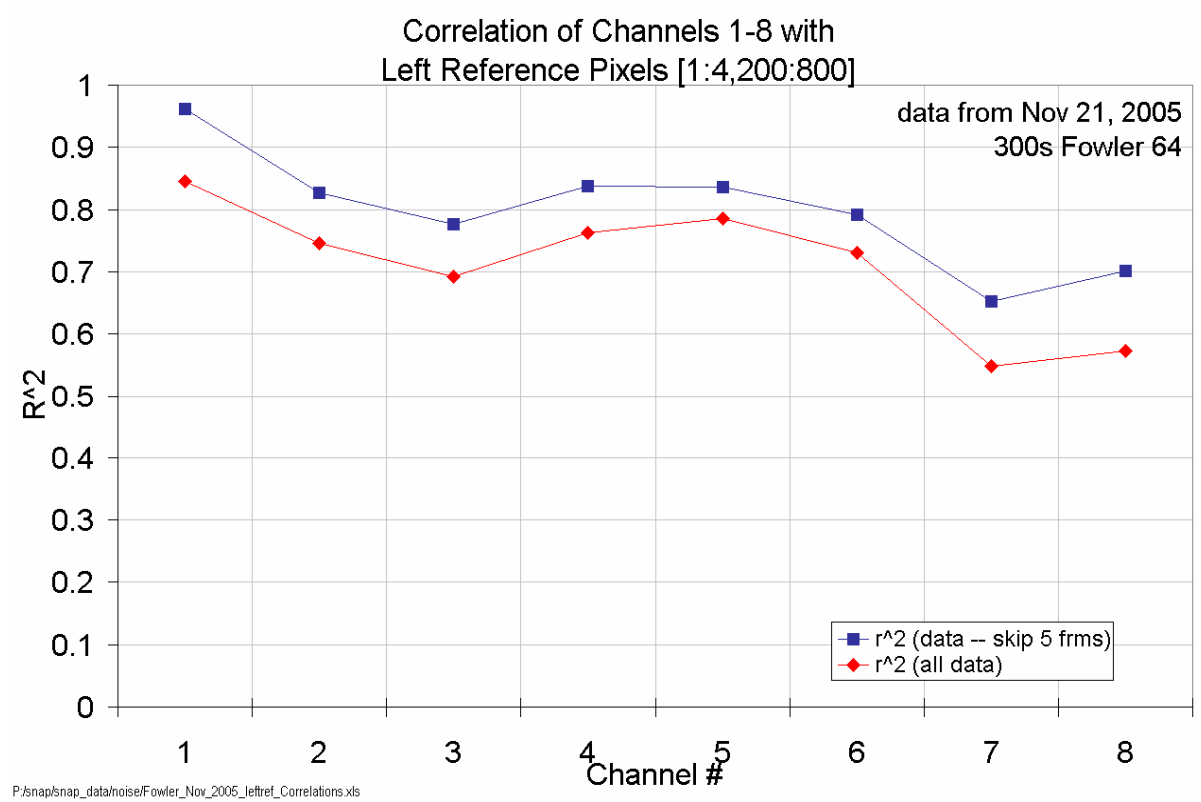

(b)

Figure 9: Correlation of noise from each of 8 channels and reference pixels in the H1RG multiplexer. (a) The H1RG has 4-pixel wide reference pixels along each edge of the detector. The noise correlation was calculated between a given readout channel (light blue or lightly shaded region inside the central part of the detector) and the average of four reference pixels on the left edge (the crosshatched region) of the H1RG. (b) Strong correlations are seen. In contrast to that observed for HgCdTe detectors, ${ }^{3}$ reference pixel subtraction does not appear to have a major impact on reducing detector noise. Data not shown as the results are identical to the InGaAs Fowler-N curves in Figure 8. 


\subsection{Persistence}

Persistence of the InGaAs detector was measured by first taking four frames of data while illuminating the detector with a $1300 \mathrm{~nm}$ monochromator output. A $250 \mathrm{sec}$ integration time was used to obtain a signal of $\sim 80 \%$ of full well. A hole mask was used to help visualize the persistence in the image data. At the conclusion of the four frames, the light source was turned off and additional images were taken with $1 \mathrm{sec}$ integration time, every $10 \mathrm{sec}$ for $480 \mathrm{sec}$, all with the hole mask removed. The resulting data is shown in Figure 10. The persistence is seen to decay to $<0.1 \%$ of the original signal level almost instantaneously, with a subsequent, well-behaved, $55 \mathrm{sec}$ decay constant. Comparable data for $\mathrm{HgCdTe}$ detectors result in longer decay constants than observed here for InGaAs.

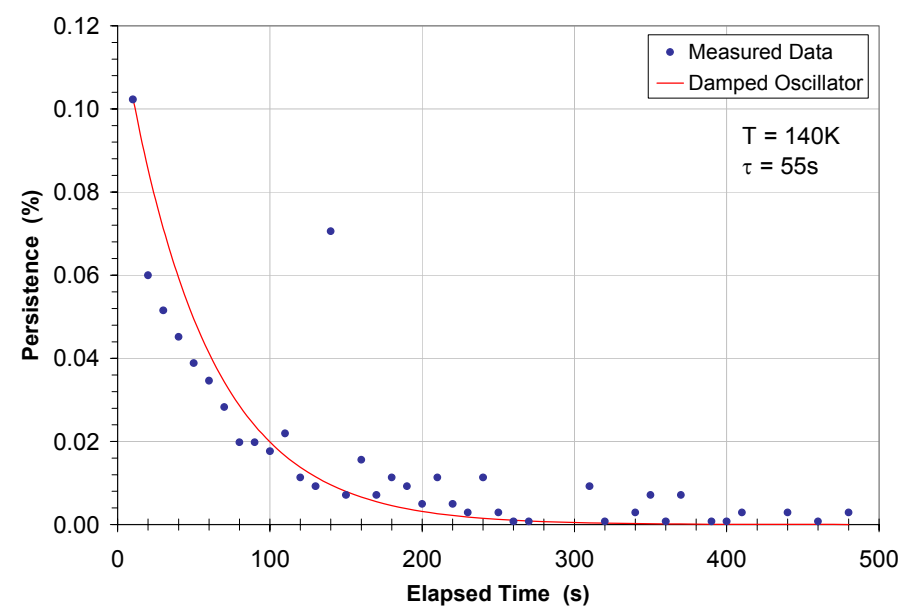

Figure 10: $140 \mathrm{~K}$ persistence data for an InGaAs focal plane array. The initial illumination level was $\sim 80 \%$ of full well.

\section{CONCLUSIONS}

Existing InGaAs detector technology (4-year old detectors) are well behaved and meet SNAP dark current goals. Noise is as good, if not better noise than existing $1.7 \mathrm{um} \mathrm{HgCdTe}$, but higher than those being developed in the SNAP R\&D program. There is a shift of the cut-off frequency from 1.7 to $1.6 \mathrm{um}$ as one goes from room temperature to $140 \mathrm{~K}$. It thus appears that existing InGaAs focal planes can meet the stringent science performance criteria. However, the improvements in $1.7 \mathrm{um} \mathrm{HgCdTe}$ arrays initiated by the SNAP R\&D effort and their availability in $2 \mathrm{k}$ x $2 \mathrm{k}$ format makes them the preferred baseline option.

\section{ACKNOWLEDGEMENTS}

The effort described herein was supported by funds from the JPL STI program. The detector development and procurement was sponsored by the United States Department of Energy under contract No. DE-AC02-05CH11231.

\section{REFERENCES}

1. M.G. Brown, Development of NIR Detectors and Science Driven Requirements for SNAP, Astronomical Telescopes and Instrumentation 2006, Proc. SPIE 6265 paper113.

2. M. Schubnell et al., Near infrared detectors for SNAP, Astronomical Telescopes and Instrumentation 2006, Proc. SPIE 6276 paper 29.

3. R. Smith, et al., Noise and zero-point drifts in 1.7um cutoff detectors for SNAP, Astronomical Telescopes and Instrumentation 2006, Proc. SPIE 6276 paper 30.

4. R.J. van der A et al., Low noise InGaAs infrared (1.0-2.um) focal plane arrays for SCIAMACHY, SPIE Proceedings V2957, 1997, p54. 
5. T. Martin, et al., 640-512 InGaAs focal plane array camera for visible and SWIR imaging, Infrared Technology and Applications XXXI, edited by Bjørn F. Andresen,Gabor F. Fulop, Proc. of SPIE Vol. 5783 (SPIE, Bellingham, WA, $2005, \mathrm{p} 12$.

6. A. Hoffman et al., Megapixel InGaAs Arrays for Low Background Applications, Infrared Technology and Applications XXXI, edited by Bjørn F. Andresen,Gabor F. Fulop, Proc. of SPIE Vol. 5783 (SPIE, Bellingham, WA, $2005, \mathrm{p} 32$.

7. Software provided by Marco Bonati and Roger Smith of the California Institute of Technology, Department of Astronomy, Optical Observatories group.

8. B. Pain and B. Hancock, Accurate Measurement of Conversion Gain and Quantum Efficiency in CMOS Imagers, IS\&T/SPIE 15th. Annual Symposium, Sensors, Cameras, and Systems for Scientific/Industrial Applications Conference 5017A, January 22, 2003.

9. A. Moore et al., Proc. SPIE V. 5167 (2003).; A. Moore et al. QE overestimation and Deterministic Crosstalk Resulting from Interpixel Capacitance, Optical Engineering, V 45-7.; G. Finger, et al., Conversion Gain and Interpixel Capacitance of CMOS Hybrid Focal Plane Arrays, Proc. Scientific Detectors for Astronomy 2005, Eds. J.E. Beletic, P. Amico, Springer Astrophysics and Space Sciences Library, V336, 477-490.

10. Autocorrelation calculation performed by M. Bonati of California Institute of Technology, Department of Astronomy, Optical Observatories group. 